\title{
Containing intense laser light in circular cavity with magnetic trap door
}

\author{
X. H. Yang, ${ }^{1, a)}$ W. Yu, ${ }^{2}$ M. Y. Yu, ${ }^{3,4, b)}$ H. Xu, ${ }^{5}$ Y. Y. Ma, ${ }^{1,8}$ Z. M. Sheng, ${ }^{6,7,8}$ H. B. Zhuo, ${ }^{1,8}$ \\ Z. Y. Ge, ${ }^{1}$ and F. Q. Shao ${ }^{1}$ \\ ${ }^{1}$ College of Science, National University of Defense Technology, Changsha 410073, China \\ ${ }^{2}$ Shanghai Institute of Optics and Fine Mechanics, Chinese Academy of Sciences, Shanghai 201800, China \\ ${ }^{3}$ Institute for Fusion Theory and Simulation, Zhejiang University, Hangzhou 310027, China \\ ${ }^{4}$ Institut für Theoretische Physik I, Ruhr-Universität Bochum, D-44780 Bochum, Germany \\ ${ }^{5}$ School of Computer Science, National University of Defense Technology, Changsha 410073, China \\ ${ }^{6}$ Key Laboratory for Laser Plasmas (Ministry of Education), Department of Physics and Astronomy, \\ Shanghai Jiao Tong University, Shanghai 200240, China \\ ${ }^{7}$ SUPA, Department of Physics, University of Strathclyde, Glasgow G4 ONG, United Kingdom \\ ${ }^{8}$ IFSA Collaborative Innovation Center, Shanghai Jiao Tong University, Shanghai 200240, P. R. China
}

(Received 18 December 2016; accepted 4 March 2017; published online 17 March 2017)

\begin{abstract}
It is shown by particle-in-cell simulation that intense circularly polarized (CP) laser light can be contained in the cavity of a solid-density circular Al-plasma shell for hundreds of light-wave periods before it is dissipated by laser-plasma interaction. A right-hand CP laser pulse can propagate with almost no reflection and attenuation into the cavity through a highly magnetized overdense $\mathrm{H}$-plasma slab filling the entrance hole. The entrapped laser light is then multiply reflected at the inner surfaces of the slab and shell plasmas, slowly losing energy to the latter. Compared to that of the incident laser, the frequency is only slightly broadened and the wave vector slightly modified by the appearance of weak nearly isotropic and homogeneous fluctuations. Published by AIP Publishing.

[http://dx.doi.org/10.1063/1.4978695]
\end{abstract}

Recently, intense laser interaction with plasma has been widely studied in connection with applications such as inertial confinement fusion $^{1}$ and laser-driven particle acceleration, ${ }^{2-4}$ as well as novel light sources. ${ }^{5,6}$ Particle-in-cell (PIC) simulations and theoretical studies ${ }^{7,8}$ have shown that isolated intense light pulses on the scale of the laser wavelength can be self-consistently trapped in plasmas as solitons. Trapping of electromagnetic (EM) waves in plasma can also be from excitation of surface plasma waves, ${ }^{9-11}$ beam-plasma, and/or parametric instabilities. ${ }^{12,13}$ In these cases, there is much loss of the laser energy to the plasma. On the other hand, there has also been considerable research on trapping and localizing relatively weak light in atomic media or photonic structures. ${ }^{14-17}$

Intense magnetic fields of $10^{3}-10^{5} \mathrm{~T}$ and above are ubiquitous in the cosmic environment. ${ }^{18}$ Strong magnetic fields can also be self-generated during ultraintense-laser interaction with matter by compression of seeded magnetic fields, ${ }^{19-21}$ baroclinic effect, ${ }^{22}$ high-current electron beams, ${ }^{23,24}$ etc. In the presence of kilotesla magnetic fields, the electron gyrofrequency $\omega_{c}$ can be comparable to or even larger than the laser frequency $\omega_{L}$. In this case, a right-hand circularly polarized (RHCP) laser can propagate deep into an overdense plasma along an intense embedded magnetic field without encountering cutoff or resonance. ${ }^{25,26}$ Moreover, transmission of RHCP laser into dense plasma can be controlled by an intense magnetic pulse in the plasma. ${ }^{27}$

In this Letter, we propose a scheme for trapping intense light in a hollow shell of high density plasma by sending a RHCP laser light pulse through a strongly magnetized slab that serves as a transparent trap door. The laser pulse can propagate through the slab with almost no reflection. Once

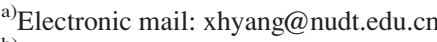

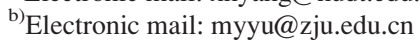

the entire laser pulse (which can be suitably long) is inside the shell cavity, the magnetic field in the slab is turned off, so that the light becomes contained. It is then multiply reflected by the unmagnetized shell plasma or propagates along its surface. As a result, the light can survive for hundreds of light-wave periods until all its energy is absorbed by the slab and shell plasma.

The optical properties of a linear medium can be described by its refractive index $N=c k_{L} / \omega_{L}=\varepsilon^{1 / 2},{ }^{25}$ where $k_{L}=2 \pi / \lambda_{L}, \omega_{L}$, and $c$ are the wave number, frequency, and vacuum speed of light, and $\varepsilon$ is the dielectric constant of the medium. For convenience, in the following we shall normalize the plasma density $n_{e}$ by the critical density $n_{c}=m_{e} \omega_{L}^{2} /$ $4 \pi e^{2}$, where $e$ and $m_{e}$ are the electron charge and mass, respectively. Accordingly, for unmagnetized plasma we have $\varepsilon=1-\omega_{p}^{2} / \omega_{L}^{2}=1-n_{e}$. Thus, light can only propagate in underdense plasmas, where $0<\varepsilon<1$. For magnetized plasma with the external magnetic field $\boldsymbol{B}_{0}$ (normalized by $\left.m_{e} c \omega_{L} / e\right)$ along the laser propagation direction, the dielectric constant can be written as $\varepsilon_{B}=1-n_{e} /\left(1 \pm B_{0}\right),{ }^{25,26}$ where the plus and minus signs correspond to the so-called $\mathrm{L}$ and $\mathrm{R}$ waves, respectively. ${ }^{25}$ Thus, for the $\mathrm{R}$ wave $\varepsilon_{B}$ is always larger than unity if $B_{0}>1$, so that the plasma is transparent at any density. In contrast, the $\mathrm{L}$ wave has a cutoff density at $n_{L}=1+B_{0}$, where the light will be reflected.

The proposed trapping scheme is illustrated in Fig. 1(a). The circular plasma shell has on its left wall a small section replaced by a highly magnetized overdense flat slab that serves as a trap door for the incident laser pulse. In order to see the function of the slab, it is instructive to first recall the onedimensional linear theory of light transmission through a thin highly magnetized high-density plasma layer, as shown in Fig. 1(b). The total wave electric field $E_{y}(x)$ in each of the three regions can be written as ${ }^{28,29} E_{i} \exp \left(-i k_{L} x\right)+E_{1} \exp \left(i k_{L} x\right)$ 
(a)

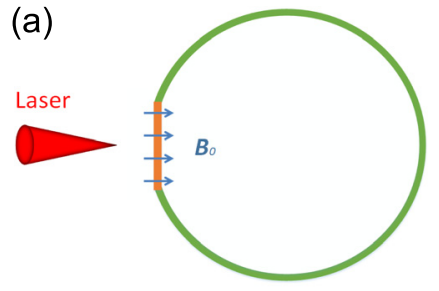

(c)

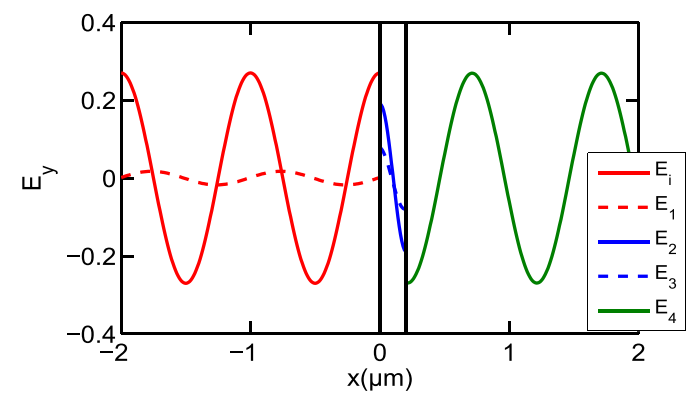

FIG. 1. (a) The proposed scheme. The entrance hole on the left shell wall is fitted with a flat overdense slab, which is strongly magnetized until the laser pulse has entered the cavity, and (b) one-dimensional model for the slab used in illustrating the $\mathrm{R}$ wave propagation. (c) Analytical solution for linear propagation of the $\mathrm{R}$ wave through the slab. Here and in the following figures, $E_{y}$ is normalized by $m_{e} c \omega_{L} / e$, corresponding to $3.22 \times 10^{12} \mathrm{~V} / \mathrm{m}$. Laser propagation and external magnetic field are along the $+x$ direction.

for $x<0, E_{2} \exp \left(-i k_{s} x\right)+E_{3} \exp \left(i k_{s} x\right)$ for $0<x<d$, and $E_{4} \exp \left[-i k_{L}(x-d)\right]$ for $x>d$, where $E_{i}$ and $E_{1}, E_{2}, E_{3}$, and $E_{4}$ are the amplitudes of the laser light incident on and reflected from the front surface of the slab, transmitted into the slab, reflected from the back surface of the slab, and transmitted into the vacuum, respectively, $k_{s}=\varepsilon_{B}^{1 / 2} k_{L}$ is the wave number in the slab. Applying the boundary conditions that at each of the boundaries (the total) $E_{y}$ and $\partial_{x} E_{y}$ should be continuous, one obtains after some algebra

$$
\begin{aligned}
& E_{1}=\frac{-i 2 R E_{0} \sin \left(k_{s} d\right)}{R^{2} e^{i k_{s} d}-e^{-i k_{s} d},} \\
& E_{2}=\frac{2 R E_{0}}{R\left(N_{s}+1\right)-\left(N_{s}-1\right) e^{-i 2 k_{s} d}}, \\
& E_{3}=\frac{2 E_{0}}{R\left(N_{s}+1\right) e^{i 2 k_{s} d}-\left(N_{s}-1\right)}, \\
& E_{4}=\frac{4 N_{s} E_{0}}{\left(N_{s}+1\right)^{2} e^{i k_{s} d}-\left(N_{s}-1\right)^{2} e^{-i k_{s} d}},
\end{aligned}
$$

where $N_{s}=\varepsilon_{B}^{1 / 2}$ and $R=\left(N_{s}+1\right) /\left(N_{s}-1\right)$. Additional internal reflections are negligible. Fig. 1(c) shows the profile of the electric field obtained from Eq. (1) for $n_{e}=20 n_{c}$, $d=0.2 \mu \mathrm{m}, B_{0}=5$, and $E_{i}=0.27$ (the same as the corresponding parameters used in the PIC simulation below). We see that the reflected light $\left(E_{1}=0.017\right)$ is only $6 \%$ of that $\left(E_{i}=0.27\right)$ of the incident, and the transmitted light $\left(E_{4}=0.269\right)$ is very close to the latter.

The relativistic 2D3V PIC simulation code $\mathrm{EPOCH}^{30}$ is used to investigate our scheme. The shell is made of $\mathrm{Al}$ plasma (ion mass $m_{A l}=26.98 m_{p}$, where $m_{p}=1836 m_{e}$ is the proton mass) with charge $Z e=10 e$ and ion density $50 n_{c}$. Its inner radius and thickness are $14.8 \mu \mathrm{m}$ and $0.2 \mu \mathrm{m}$, respectively. The slab consists of hydrogen plasma with density $20 n_{c}$. Its height and thickness are $10 \mu \mathrm{m}$ and $0.2 \mu \mathrm{m}$, respectively. The center of the shell cavity is located at $(x, y)=(20,20) \mu \mathrm{m}$, so that the left front of the slab is at $x=5.86 \mu \mathrm{m}$. An external magnetic field of strength $B_{0}=5$ (corresponding to $5.36 \times 10^{4} \mathrm{~T}$ in dimensional units) along the laser axis is embedded in the slab plasma, which is shut down after the laser pulse injection is complete. To account for laser-plasma interaction induced plasma expansion, the extent of $\boldsymbol{B}_{0}$ is slightly wider than the slab. The initial temperature of the slab and shell is $100 \mathrm{eV}$. The simulation box is $40 \mu \mathrm{m} \times 40 \mu \mathrm{m}$ with $4000 \times 4000$ cells. Each cell contains 50 macro particles per species. At $t=0$, a RHCP laser pulse of wavelength $\lambda=1 \mu \mathrm{m}$ and intensity $I_{0}=2 \times 10^{17} \mathrm{~W} / \mathrm{cm}^{2}$ (or laser parameter $a_{L}=e E_{L} / m_{e} c \omega_{L}=0.27$, where $E_{L}$ is the peak laser electric field) enters from the left side $(x=0)$ of the simulation box and is focused on the slab at $x=5.86 \mu \mathrm{m}$. Its front rises with a Gaussian profile for $17 \mathrm{fs}$ to the peak intensity and remains constant for $150 \mathrm{fs}$. The transverse profile of the laser pulse is Gaussian, with spot radius $2 \mu \mathrm{m}$.

Figure 2 shows the wave electric field $E_{y}$, magnetic field $B_{z}$, and axial Poynting vector $S_{x}$ at $t=100 \mathrm{fs}$. We see that the laser pulse can easily enter the overdense slab into the cavity. It propagates through the slab almost without reflection, and the amplitude of the transmitted laser in the vacuum is very close to that of the incident laser, in good agreement with the theoretical analysis (see Fig. 1(c)). One can see from Fig. 2 for $t=100 \mathrm{fs}$ that there is little energy loss by the laser as it passed the slab. The axial Poynting flux is everywhere positive except at the shell-slab boundary, where some light is scattered.

Reflections from the cavity wall and wave interference lead to restructuring of the EM fields in the cavity as well as loss of wave energy to the slab and shell plasmas. Figure 3 for $t=350$ fs shows the transverse electric field $E_{y}$, magnetic field $B_{z}$, and EM field energy density $\mathcal{W}=\frac{1}{2}\left(\epsilon_{0}|\boldsymbol{E}|^{2}\right.$ $\left.+\mu_{0}^{-1}|\boldsymbol{B}|^{2}\right)$, where $\epsilon_{0}$ and $\mu_{0}$ are the free-space permittivity
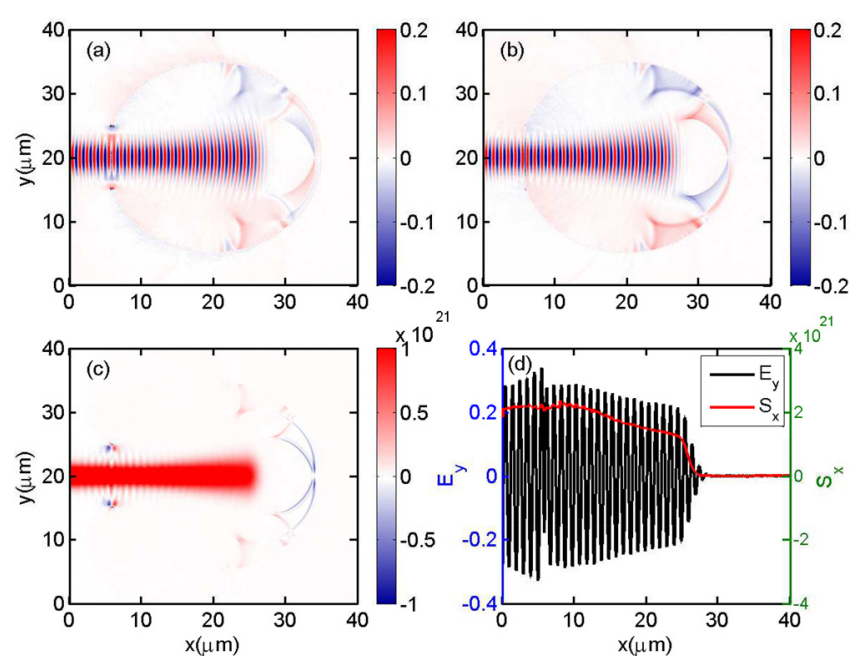

FIG. 2. Distribution of the wave (a) electric $\left(E_{y}\right)$ and (b) magnetic $\left(B_{z}\right)$ fields, and (c) axial Poynting flux $\left(S_{x}\right)$ at $t=100$ fs. (d) $E_{y}$ and $S_{x}$ (averaged over $0.5 \mu \mathrm{m}$ around $y=20 \mu \mathrm{m}$ ) along the $x$ direction. Here and in the following figures, $B_{z}$ is normalized by $m_{e} c \omega_{L} / e$ (corresponding to $1.07 \times 10^{4} \mathrm{~T}$ ) and $S_{x}$ is in units of $\mathrm{W} / \mathrm{m}^{2}$. The highly localized $E_{y}$ and $S_{x}$ fields at the top and bottom slab-shell boundaries can be attributed to the complex laser-plasma interaction there. See also Fig. 3(c). 

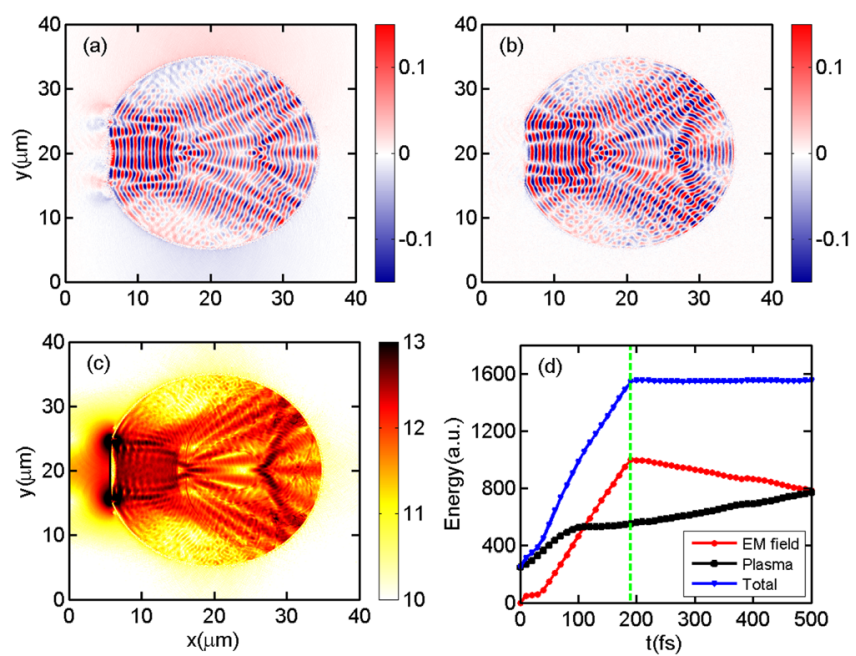

FIG. 3. Distribution of (a) $E_{y}$, (b) $B_{z}$, and (c) EM field energy density (in $\mathrm{J} / \mathrm{m}^{3}$ ) at $t=350 \mathrm{fs}$. (d) Evolution of the energies of the light waves in the shell (red circles), the plasma (including the shell and slab, black squares) and the sum of the former energies (blue triangles). The green dashed line in (d) marks the termination of the laser pulse injecting into the shell. Recall that the latter has a short (17 fs) Gaussian front, followed by a long (150 fs) flat-top tail.

and permeability, respectively. We see that light is trapped in the cavity and its structure is rather complex. Because of constructive interference of the reflecting light in the cavity, locally the magnitudes of the wave electric and magnetic fields can be up to 1.61 and 2.35, respectively, times that of the incident laser. Figure 3(d) for the evolution of the energies of the trapped light and the plasmas shows that the former increases with time until the laser pulse has completely entered the cavity (at $t \sim 190 \mathrm{fs}$, recall that the slab is $5.86 \mu \mathrm{m}$ away from the left side of the simulation box). Then, it gradually decreases. One can also see that the energy of the plasmas first increases rapidly, mainly due to light absorption by the slab electrons, which is heated and expands (not shown). Figure 3(d) also shows that after the laser pulse has completely entered the cavity at $t \sim 190 \mathrm{fs}$, energy absorption by the plasma continues at a slower rate, and the total energy of the light in the cavity and the plasma in the slab and shell is well conserved.

On the other hand, Fig. 3(d) also shows that even though transfer of light energy to the slab and shell plasmas starts as soon as the laser enters the slab, even at $t=500 \mathrm{fs}$ the trapped light still retains about $78.6 \%$ of its maximum energy, namely, that at $t \sim 190$ fs. We have not attempted to optimize the light trapping, which has to be done by trial and error adjustment of the initial parameters. It should also be possible to improve the design of the slab so that absorption of reflected light by its cavity facing side is minimized.

Figure 4 shows the spatial EM field spectra $E_{y}(\boldsymbol{k})$ and $B_{z}(\boldsymbol{k})$ at two instants after the laser pulse has fully entered the cavity. One can see that even though the spatial wave structure in the real space is rather complex, its Fourier space is relatively simple. As mentioned, initially the $\pm k_{x}$ components from the just-transmitted laser light dominate (not shown), but $k_{y}$ components also appear as reflections take place since the laser is of finite spot size and the cavity wall is curved. Figures 4(a)-4(c) show that except around $\theta \sim \pi / 2$ and $3 \pi / 2$ (apparently due to less light reflections
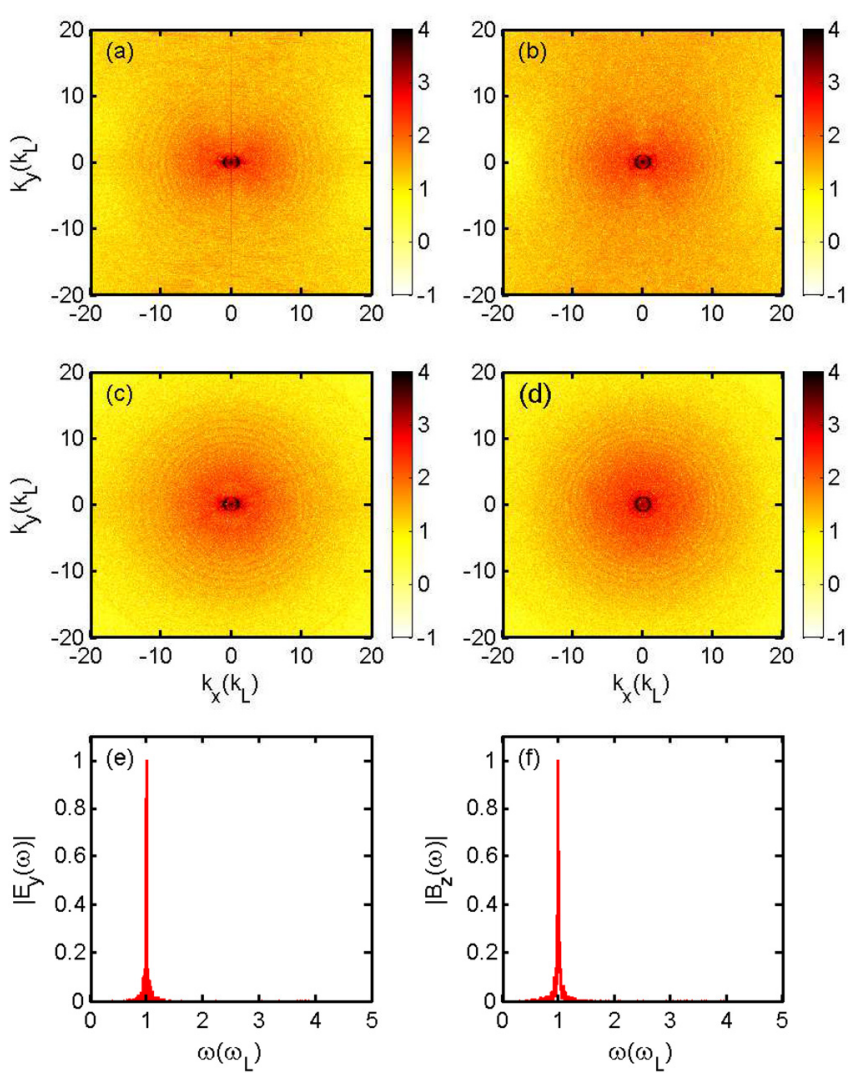

FIG. 4. Fast Fourier transform of $E_{y}$ (top row) and $B_{z}$ (bottom row) at $t=300 \mathrm{fs}$ (left column) and $400 \mathrm{fs}$ (right column). Frequency spectra $\left|E_{y}(\omega)\right|$ (e) and $\left|B_{z}(\omega)\right|$ (f) (arbitrary units) at the center ( $x=20 \mu \mathrm{m}$, $y=20 \mu \mathrm{m})$ of the cavity.

occurring around these angles in the physical space), the $|\boldsymbol{k}|=1$ mode (small dark partial circles in the figures) associated with that $\left(\boldsymbol{k}=1 \hat{e}_{x}\right)$ of the input laser remains dominant at all times. In fact, close examination shows that the $|\boldsymbol{k}|<1$ region is more highly populated around the $\pm x$ directions, as to be expected. Weak but distinguishable spatial harmonics $|\boldsymbol{k}|=2,3,4, \ldots$ can also be observed. The spectra continue to evolve with time as energy loss to the slab and shell plasma continues, but their overall profiles remain almost unchanged. In particular, they remain peaked at $|\boldsymbol{k}|$ $=1$ (except around $\theta \sim \pi / 2$ and $3 \pi / 2$ ) and $k=0$. Figures 4(e) and 4(f) show the frequency spectra $E_{y}(\omega)$ and $B_{z}(\omega)$ at the center of the cavity. At other locations in the cavity, they (not shown) are very similar. We see that the frequency spectrum is highly peaked at the incident laser frequency $\omega_{L}$, which together with the dominance of the wavelength near that of the incident laser confirms that the light of the latter is trapped without altering its basic characteristics. The slight frequency broadening can be attributed to the presence in the cavity of low-density electrons driven out by the laser-wall plasma interaction, so that the vacuum light-wave dispersion relation is slightly modified by inclusion of the plasma frequency $\omega_{p}\left(\ll k_{L} c\right)$. However, as already pointed out, we were unable to identify the EM field or Poynting vector structures with that of an eigenmode solution of the vacuum wave equation in circular geometry, ${ }^{31}$ even though the trapped light has quite well defined frequency and wavelength, as well as $E_{y}(\omega, \boldsymbol{k})$ and $B_{z}(\omega, \boldsymbol{k})$ relationship. The reason could be that there is insufficient time to form an 

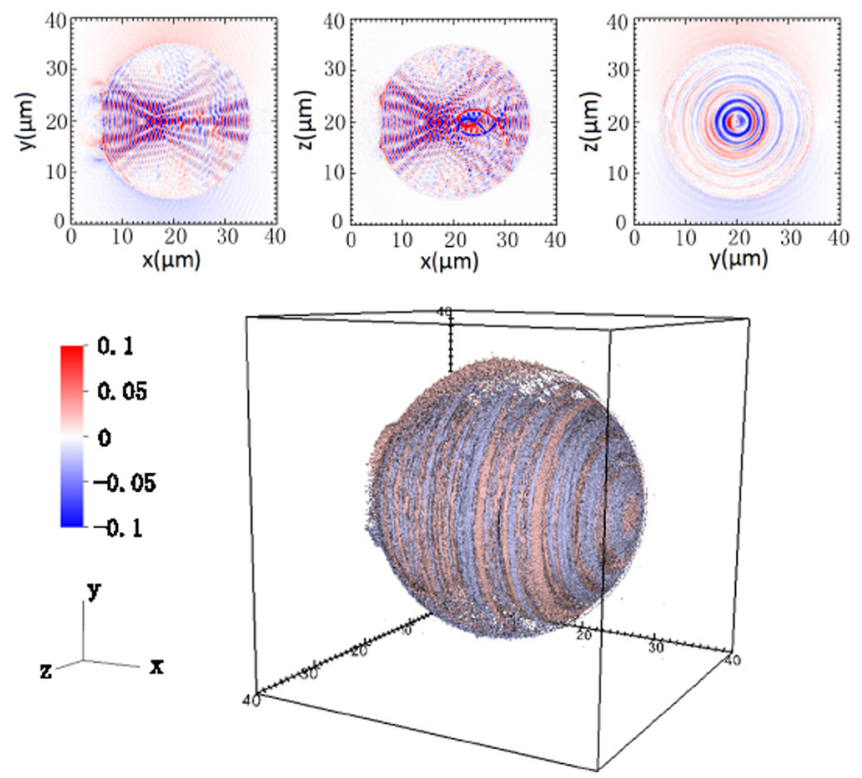

FIG. 5. Trapped light in the spherical cavity. (a)-(c) Distribution of the wave electric field $E_{y}$ in the $x-y, x-z$, and $y-z$ planes, respectively, from the $3 \mathrm{D}$ simulation at $t=340 \mathrm{fs}$, and (d) a $3 \mathrm{D}$ view.

eigenmode, the cavity is not really circularly symmetric because of the slab, and/or the wave-plasma interaction at the slab and cavity wall cannot be ignored. Furthermore, one can see in Figs. 4(a)-4(d) that the region near $k=0$ is also enhanced, which can be attributed to the multiple light reflections at the slab and shell plasma boundaries, where $\boldsymbol{k}$ changes sign.

In order to confirm that our scheme is also applicable in the more realistic spherical geometry, we have also carried out simulations for laser light trapping in a spherical shell. The simulation box has $1000 \times 800 \times 800$ cells, with each cell containing 36 macro particles of each species. The interaction parameters are the same as those for the preceding $2 \mathrm{D}$ simulation, except that in order to limit the computational expense the electron density of the cavity wall is now $200 n_{c}$. Figure 5 shows the distribution of wave electric field at $t=340 \mathrm{fs}$. We see that the RHCP laser light has propagated through the magnetized slab and is well trapped in the cavity. We note that the profiles of the wave electric field in the relevant (i.e., the $x-y$ and $x-z$ ) planes are also quite similar to those of the $2 \mathrm{D}$ results.

In conclusion, we have considered a scheme for containing intense light in the cavity of a solid-density Al plasma shell. PIC simulations demonstrate that the laser light can enter the cavity with the help of an axial magnetic field embedded in an overdense $H$ slab covering a hole in the shell. Inside the cavity the light wave is reflected as well as partially absorbed by the slab and shell plasma. Because of the multiple reflections, the wave frequency is slightly broadened but remains close to that of the incident laser. The wave vector, originally only along the laser axis, also acquires new directions and weak spatial harmonics are generated, which then become azimuthally isotropic except around $\theta \sim \pi / 2$ and $3 \pi / 2$. However, the wavelength of the incident laser still remains the dominating spatial scale. Because of energy transfer to the slab and shell, the trapped light continues to decay until it vanishes. The results here may be useful for understanding storage of intense light, as well as interpretation of phenomenon associated with highly localized light in nature. ${ }^{32,33}$

We would like thank S. H. Luan for useful discussions. This work was supported by the NNSFC (11305264, 11275269, 11374262, 11375265, 11475260, and 91230205), the Open Fund of the State Key Laboratory of High Field Laser Physics at SIOM, and the Research Program of NUDT.

${ }^{1}$ J. Lindl, O. Landen, J. Edwards, E. Moses, and NIC Team, Phys. Plasmas 21, 020501 (2014).

${ }^{2}$ A. Macchi, M. Borghesi, and M. Passoni, Rev. Mod. Phys. 85, 751 (2013). ${ }^{3}$ X. H. Yang, W. Yu, H. Xu, H. B. Zhuo, Y. Y. Ma, D. B. Zou, T. P. Yu, Z. Y. Ge, Y. Yin, F. Q. Shao, and M. Borghesi, Phys. Plasmas 21, 063105 (2014).

${ }^{4}$ X. H. Yang, H. B. Zhuo, Y. Y. Ma, H. Xu, T. P. Yu, D. B. Zou, Z. Y. Ge, B. B. Xu, Q. J. Zhu, F. Q. Shao, and M. Borghesi, Plasma Phys. Controlled Fusion 57, 025011 (2015).

${ }^{5}$ Zs. Lécz and A. Andreev, Phys. Rev. E 93, 013207 (2016).

${ }^{6}$ J. Vieira, R. M. G. M. Trines, E. P. Alves, R. A. Fonseca, J. T. Mendonca, R. Bingham, P. Norreys, and L. O. Silva, Phys. Rev. Lett. 117, 265001 (2016).

${ }^{7}$ N. M. Naumova, S. V. Bulanov, T. Zh. Esirkepov, D. Farina, K. Nishihara, F. Pegoraro, H. Ruhl, and A. S. Sakharov, Phys. Rev. Lett. 87, 185004 (2001).

${ }^{8}$ M. Y. Yu, P. K. Shukla, and K. H. Spatschek, Phys. Rev. A 18, 1591 (1978).

${ }^{9}$ L. Stenflo, Phys. Scr. 1996, 59 (1996).

${ }^{10}$ M. I. Bakunov and A. V. Maslov, Phys. Rev. Lett. 79, 4585 (1997).

${ }^{11}$ N. Kumar and V. K. Tripathi, Phys. Plasmas 14, 103108 (2007).

${ }^{12}$ P. Kaw, G. Schmidt, and T. Wilcox, Phys. Fluids 16, 1522 (1973).

${ }^{13}$ V. P. Silin and A. N. Starodub, Zh. Eksp. Teor. Fiz. 73, 1379 (1977).

${ }^{14}$ L. V. Hau, S. E. Harris, and C. H. Behroozi, Nature 397, 594 (1999).

${ }^{15}$ M. Fleischhauer and M. D. Lukin, Phys. Rev. Lett. 84, 5094 (2000).

${ }^{16}$ T. Tanabe, M. Notomi, E. Kuramochi, A. Shinya, and H. Taniyama, Nat. Photonics 1, 49 (2007).

${ }^{17}$ P. N. Melentiev, A. E. Afanasiev, A. A. Kuzin, A. S. Baturin, and V. I. Balykin, Opt. Lett. 38, 2274 (2013).

${ }^{18}$ V. Canuto and H. Y. Chiu, Space Sci. Rev. 12, 3 (1971).

${ }^{19}$ J. P. Knauer, O. V. Gotchev, P. Y. Chang, D. D. Meyerhofer, O. Polomarov, R. Betti, J. A. Frenje, C. K. Li, M. J. E. Manuel, R. D. Petrasso, J. R. Rygg, and F. H. Séguin, Phys. Plasmas 17, 056318 (2010).

${ }^{20}$ P. Y. Chang, G. Fiksel, M. Hohenberger, J. P. Knauer, R. Betti, F. J. Marshall, D. D. Meyerhofer, F. H. Séguin, and R. D. Petrasso, Phys. Rev. Lett. 107, 035006 (2011).

${ }^{21}$ H. Yoneda, T. Namiki, A. Nishida, R. Kodama, Y. Sakawa, Y. Kuramitsu, T. Morita, K. Nishio, and T. Ide, Phys. Rev. Lett. 109, 125004 (2012).

${ }^{22}$ U. Wagner, M. Tatarakis, A. Gopal, F. N. Beg, E. L. Clark, A. E. Dangor, R. G. Evans, M. G. Haines, S. P. D. Mangles, P. A. Norreys, M. S. Wei, M. Zepf, and K. Krushelnick, Phys. Rev. E 70, 026401 (2004).

${ }^{23}$ S. Fujioka, Z. Zhang, K. Ishihara, K. Shigemori, Y. Hironaka, T. Johzaki, A. Sunahara, N. Yamamoto, H. Nakashima, T. Watanabe, H. Shiraga, H. Nishimura, and H. Azechi, Sci. Rep. 3, 1170 (2013).

${ }^{24} \mathrm{Ph}$. Korneev, E. d'Humières, and V. Tikhonchuk, Phys. Rev. E 91, 043107 (2015).

${ }^{25}$ F. F. Chen, Introduction to Plasma Physics and Controlled Fusion, 2nd ed. (Springer, New York, 2006).

${ }^{26}$ X. H. Yang, W. Yu, H. Xu, M. Y. Yu, Z. Y. Ge, B. B. Xu, H. B. Zhuo, Y. Y. Ma, F. Q. Shao, and M. Borghesi, Appl. Phys. Lett. 106, 224103 (2015).

${ }^{27}$ G. J. Ma, W. Yu, M. Y. Yu, S. X. Luan, and D. Wu, Phys. Rev. E 93, 053209 (2016).

${ }^{28}$ J. A. Kong, Electromagnetic Wave Theory (Wiley, New York, 1990).

${ }^{29}$ R. W. Scharstein, IEEE Trans. Educ. 35, 170 (1992).

${ }^{30}$ T. D. Arber, K. Bennett, C. S. Brady, A. Lawrence-Douglas, M. G. Ramsay, N. J. Sircombe, P. Gillies, R. G. Evans, H. Schmitz, A. R. Bell, and C. P. Ridgers, Plasma Phys. Controlled Fusion 57, 113001 (2015).

${ }^{31}$ M. J. Padgett and L. Allen, Opt. Commun. 121, 36 (1995).

${ }^{32}$ M. Stenhoff, Ball Lightning: Unsolved Problem in Atmospheric Physics (Kluwer, New York, 1999).

${ }^{33}$ H. C. Wu, Sci. Rep. 6, 28263 (2016). 